\title{
Dipolar and Quadrupolar Magnetic Field Evolution over Solar Cycles 21, 22, and 23
}

\author{
M. L. DeRosa ${ }^{1}$, A. S. Brun ${ }^{2}$ and J. T. Hoeksema ${ }^{3}$ \\ ${ }^{1}$ Lockheed Martin Solar and Astrophysics Laboratory, 3251 Hanover St. B/252, Palo Alto, CA \\ 94304 USA \\ email: derosa@lmsal.com \\ ${ }^{2}$ Laboratoire AIM Paris-Saclay, CEA/Irfu Université Paris-Diderot CNRS/INSU, F-91191 \\ Gif-sur-Yvette, France \\ ${ }^{3}$ Hansen Experimental Physics Laboratory, Stanford University, 466 Via Ortega, Stanford, \\ CA 94305 USA
}

\begin{abstract}
Time series of photospheric magnetic field maps from two observatories, along with data from an evolving surface-flux transport model, are decomposed into their constituent spherical harmonic modes. The evolution of these spherical harmonic spectra reflect the modulation of bipole emergence rates through the solar activity cycle, and the subsequent dispersal, shear, and advection of magnetic flux patterns across the solar photosphere. In this article, we discuss the evolution of the dipolar and quadrupolar modes throughout the past three solar cycles (Cycles 21-23), as well as their relation to the reversal of the polar dipole during each solar maximum, and by extension to aspects of the operation of the global solar dynamo.
\end{abstract}

Keywords. Sun: magnetic fields

\section{Introduction}

Understanding the solar dynamo is one of the longstanding problems in the field of solar physics. Much of what we know about the solar dynamo arises from observations of the patterns of magnetic flux that emerge onto the photosphere and their subsequent evolution (Hathaway 2010), combined with numerical modeling efforts that focus on gaining a theoretical perspective on the interplay between the large-scale flows, the tachocline and upper shearing layers, convection, and magnetic fields within the solar interior (Charbonneau 2005; Browning et al. 2006). The most useful numerical models capture many of the observed behaviors of magnetic flux, such as (for example) the spectrum of features visible on the photosphere, or the timing and phases of dipole reversals.

As a result, long-term observations of photospheric magnetic fields over multiple solar activity cycles are extremely useful as boundary conditions for modeling, and/or as independent validation of models, or for simply gaining intuition. In this article, we take a spectral approach and analyze the solar photospheric magnetic field from the perspective of its spherical harmonic decomposition, determining the harmonic coefficients from three different time series of the photospheric magnetic field, with a special emphasis on dipolar and quadrupolar fields and their phase relationship over the past three solar cycles. The three datasets are outlined in $\S 2$, followed by a discussion of the dipolar and quardupolar modes in $\S 3$ and $\S 4$, respectively. The energy spectra are presented in $\S 5$, followed by a brief discussion and concluding remarks in $\S 6$. 


\section{Spherical Harmonic Decomposition of the Data}

Time series of maps of the radial magnetic field covering the entire solar photosphere (i.e., the full $360^{\circ}$ of longitude and $180^{\circ}$ of latitude) were decomposed into their constituent spherical harmonic modes. This process involves finding the coefficients $B_{\ell, m}(t)$ for a time series of photospheric radial magnetic field maps $B_{r}(\theta, \phi, t)$ such that

$$
B_{r}(\theta, \phi, t)=\sum_{\ell, m} B_{\ell, m}(t) Y_{\ell, m}(\theta, \phi),
$$

where the spherical harmonic functions $Y_{\ell, m}(\theta, \phi)$ are characterized by the quantum numbers degree $\ell$ and order $m$. The magnetic data originate from three different sources, as follows:

(a) Diachronic maps, sampled once per Carrington rotation (CR), were assembled from full-disk magnetograms observed by the ground-based Wilcox Solar Observatory (WSO) in Stanford, CA. The WSO maps span CR 1642-2089, containing data from 1976-May-27 through 2009-Nov-9, and are available at http://wso.stanford.edu/synopticl.html.

(b) Diachronic maps, sampled once per Carrington rotation, were assembled from fulldisk magnetograms observed by the Michelson Doppler Imager (MDI; Scherrer et al. 1995) on board the Solar and Heliospheric Observatory (SOHO) spacecraft. The MDI maps span CR 1910-2088, containing data from 1996-Jul-1 through 2009-Oct-13, and are available at http://soi.stanford.edu/magnetic/index6.html.

(c) Instantaneous synoptic maps from an evolving-flux model of the solar photosphere (Schrijver 2001) were sampled once every two weeks. The model is constructed by inserting flux from the 96-minute time series of full-disk MDI magnetograms into the model (Schrijver \& De Rosa 2003). During each 6h time step, the population of flux concentrations is advected horizontally according to empirically-based prescriptions for differential rotation, poleward meridional flows, and dispersal due to supergranulation. Neighboring flux concentrations are allowed to coalesce or partially cancel (depending on their polarities) if they become separated by a distance less than $4200 \mathrm{~km}$. The data used in this study span much of the SOHO era, ranging from 1996-Jul-1 through 2009-Dec-31, and can be downloaded using the pfss package available through SolarSoft.

\section{Evolution of Solar Dipole}

The solar dipolar magnetic field can be analyzed in terms of its polar and equatorial harmonic components. The polar component, corresponding to the $\ell=1, m=0$ spherical harmonic function and which has a mode amplitude of $B_{1,0}$, is observed to be strongest during solar minimum when there is a significant amount of magnetic flux located near the polar regions of the sun. These polar caps are the result of small amounts of trailingpolarity flux "escaping" from each active region throughout the course of a sunspot cycle, followed by their advection poleward by the meridional flow pattern. On average, flux from trailing polarities is more likely to reach the poles, from which it follows that the same amount of leading-polarity flux must cross the equator and cancel with leadingpolarity flux from the opposite hemisphere. In contrast, the equatorial dipole components, having mode amplitudes $B_{1, \pm 1}$, are strongest during solar maximum and result from the presence of active regions during the sunspot activity cycle.

Because the WSO data span multiple sunspot activity cycles, a historical perspective on the evolution of the dipole can be gained, as shown in Figures 1 and 2. For example, the upper panel of Figure 1 illustrates that the (unsigned) magnitude of the solar-minimum polar dipole is much lower at the present time, following the most recent sunspot cycle (Cycle 23), than following either of the two preceding sunspot cycles (Cycles 21 and 22). 

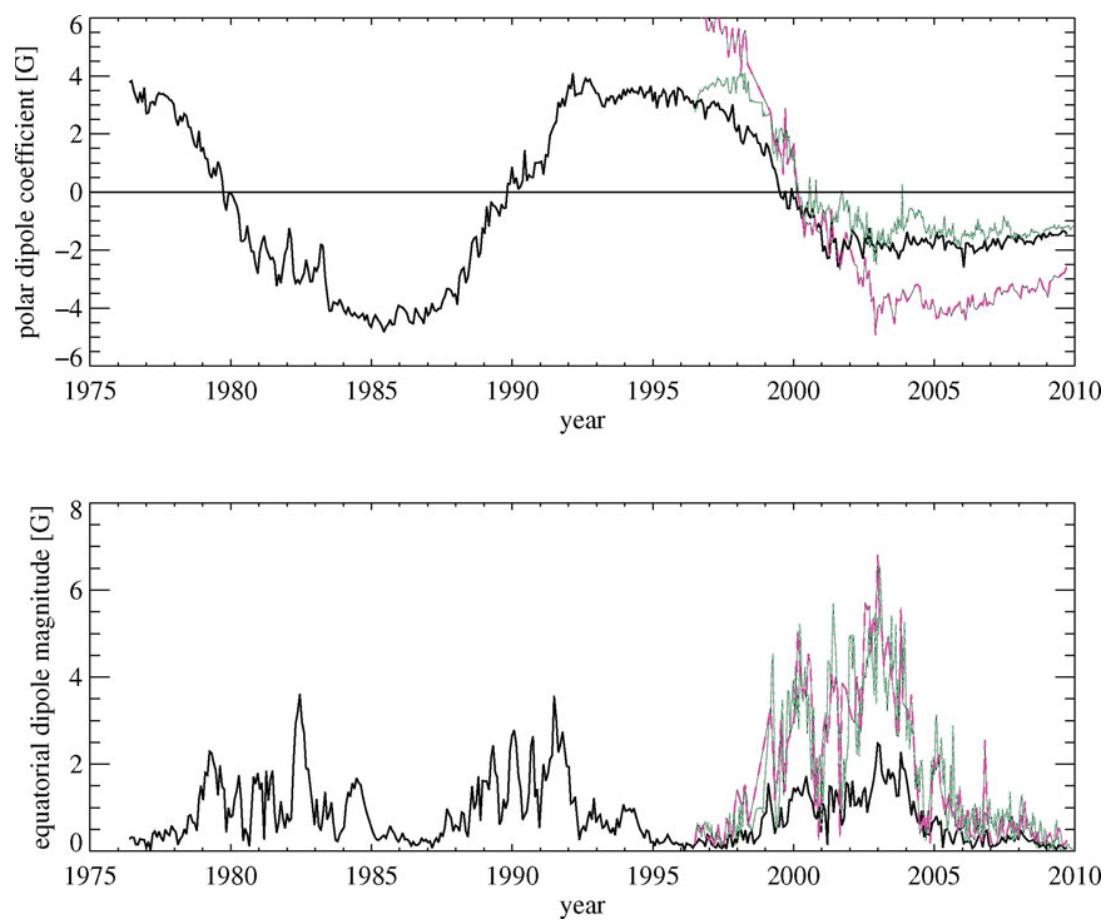

Figure 1. The temporal evolution of the (signed) polar dipole coefficient $B_{1,0}$ (upper panel), and the (unsigned) magnitude of the equatorial dipole coefficient $\left(\left|B_{1,-1}\right|^{2}+\left|B_{1,1}\right|^{2}\right)^{1 / 2}$ (lower panel) for each of the three datasets. The horizontal axis encompasses the past three sunspot cycles. In each plot, the thick solid line corresponds to the WSO maps, the dashed line corresponds to the MDI maps, and the dotted line corresponds to the evolving-flux model. [In the color version of this article (available online), the thick solid line is black, the dotted line is green, and the dashed line is magenta.]

Similarly, the equatorial dipole strength was lower during the Cycle 23 maximum than during the maxima of either Cycle 21 or 22, as shown in the lower panel of Figure 1. We infer that such cycle-to-cycle trends are likely not unusual, especially if one were to consider the variation in the sunspot index (a broad-brush measure of magnetic activity) over time as determined, for example, by the Royal Observatory of Belgium (available at http://www.sidc.be/sunspot-index-graphics/sidc_graphics.php). Interestingly, the range over which the ratio of the energies contained in the equatorial versus the polar dipole components has remained about the same over this time, as shown in the lower panel of Figure 2.

During the course of a sunspot cycle, the polar dipole reverses sign as the polar caps established during the previous cycle are eroded away and are reformed with additional opposite-polarity flux that is being advected poleward. Visualizations of the dipole axis computed from data from Cycle 23 show that this reversal process took several years to complete. During this time, and especially when $B_{1,0}$ was near zero, the reduced energy in the polar dipole was partially offset by an increase in energy in the equatorial dipole, resulting in a reduction of the total energy in all dipolar modes only by about an order of magnitude above its solar-minimum value, as shown in the upper panel of Figure 2 . When the polar dipolar component is weak, the axis of the equatorial dipolar component is observed to precess in longitude. These dynamics occur because the longitude of the dipole axis is set by the strongest active regions on the photosphere at any given time. 

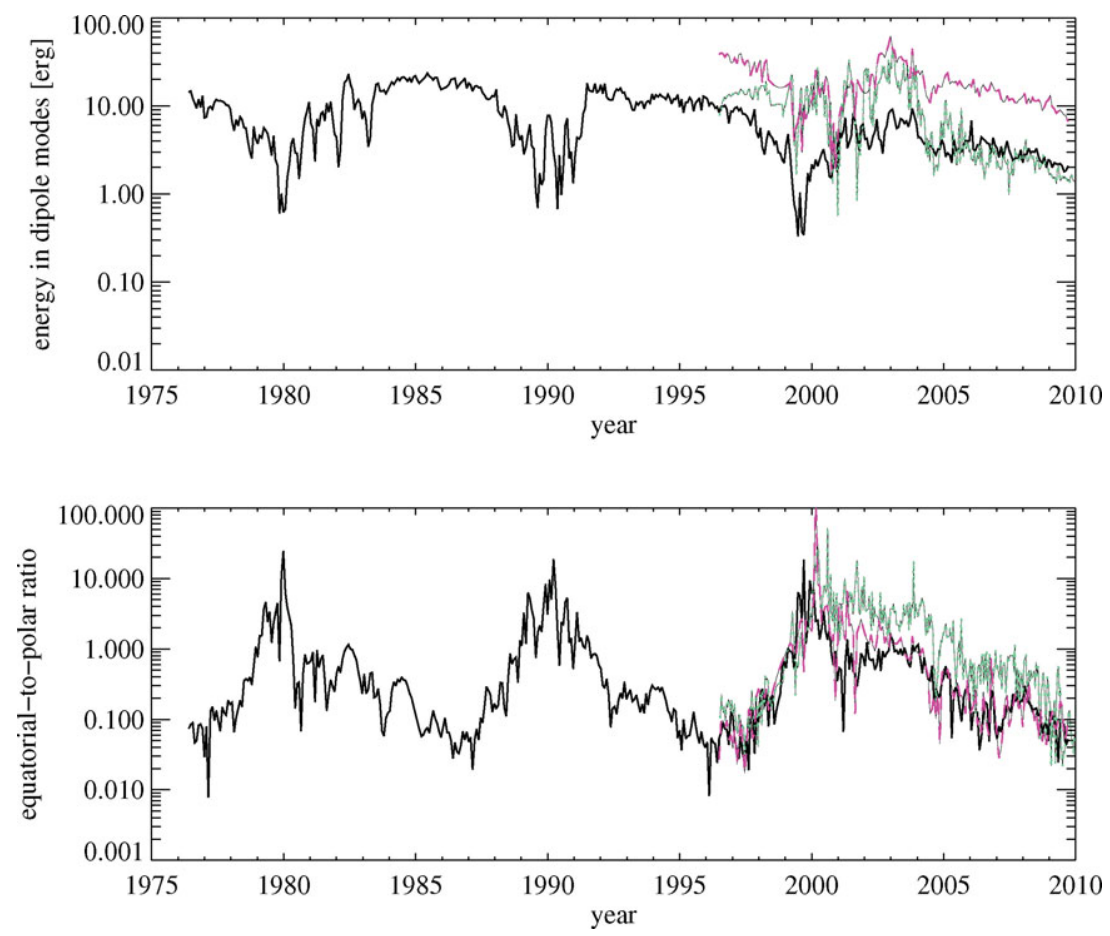

Figure 2. The temporal evolution of the total energy in the dipolar modes $\sum_{m}\left|B_{1, m}\right|^{2}$ (upper panel), and the ratio between the energy in the equatorial and polar dipole modes $\left|B_{1,0}\right|^{2} /\left(\left|B_{1,-1}\right|^{2}+\left|B_{1,1}\right|^{2}\right)$ (lower panel). The solid, dashed, and dotted lines are as in Fig. 1.

As older active regions decay and newer sources of flux appear on the photosphere, this longitude can (and does!) change in a continuous fashion in response to the ever-evolving configuration of active-region flux on the solar surface.

\section{Evolution of Solar Quadrupole}

The evolution of the energy contained in the quadrupolar $(\ell=2)$ modes exhibit much more variation than the dipolar energy, as can be seen by comparing the upper panel of Figure 2 with the upper panel of Figure 3, in which are illustrated the energy in the dipolar and quadrupolar modes, respectively. As with the equatorial dipole components, the quadrupolar modes have more power in them during maximal activity levels than during solar minima. During such times when there is a large amount of activity, it is possible for the energy in the quadrupolar modes to be greater than the energy in the dipolar modes. The ratio between these two groups of modes is shown in the lower panel of Figure 3, from which it is evident that during each of the past three sunspot cycles there have been periods of time when the quadrupolar energy has exceeded the dipolar energy by as much as a factor of 10 . An example of one such period is illustrated in the coronal field model of Figure 4, where the solar magnetic field appears to be dominated by a quadrupolar mode having an axis of symmetry in the equatorial plane oriented perpendicular to the line of sight.

\section{Energy Spectra}

One property of the spherical harmonic functions $Y_{\ell, m}(\theta, \phi)$ is that the degree $\ell$ is equal to the number of node lines (i.e., lines where $Y_{\ell, m}=0$ ) on the $r=R_{\odot}$ surface. In other words, the spatial scale represented by any harmonic mode (i.e., the distance between 

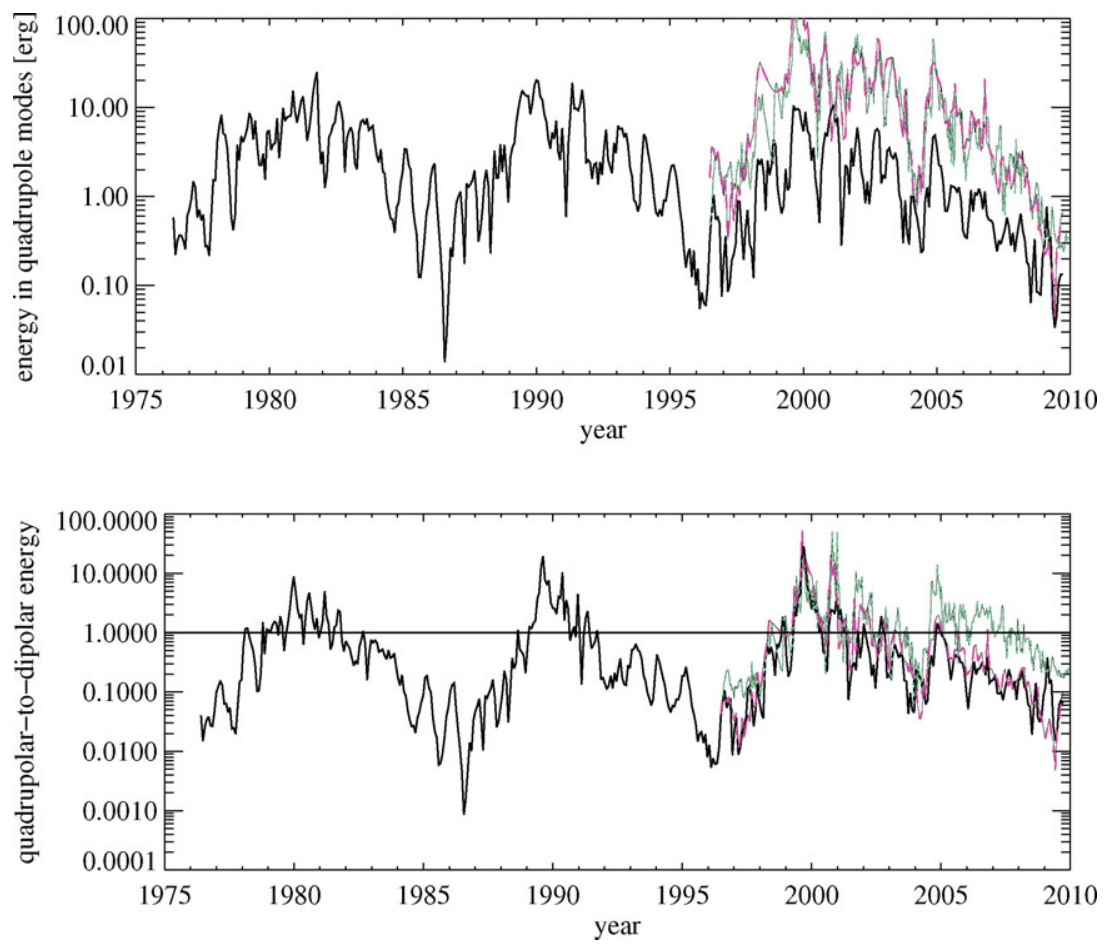

Figure 3. The temporal evolution of the total energy in the quadrupolar modes $\sum_{m}\left|B_{2, m}\right|^{2}$ (upper panel), and the ratio between the energy in the equatorial and polar dipole modes $\sum_{m}\left|B_{1, m}\right|^{2} / \sum_{m}\left|B_{2, m}\right|^{2}$ (lower panel). The solid, dashed, and dotted lines are as in Fig. 1.

neighboring node lines) is determined by its spherical harmonic degree $\ell$. As a result, the range of $\ell$ values containing the greatest amount of energy indicates of the dominant spatial scales of the magnetic field. To this end, we have averaged the power spectra from each of the three datasets both over time and over $m$, and have displayed the result in Figure 5. In the figure, it can be seen that the magnetic power spectra form a broad peak with a maximum degree occurring at about $\ell=25$. This size scale corresponds to about the same size as a typical active region, indicating that much of the magnetic energy can be found (perhaps not surprisingly) on the spatial scale of active regions.

The upper panel of Figure 5 illustrates the dependence of the energy spectra on the spatial resolution of the magnetograph. The WSO curve (the solid black line in the figure) does not show the same broad peak at $\ell=25$ as the curves from the other datasets (both of which utilize MDI data). This is because the significantly lower spatial resolution of the WSO magnetograph (which has $180^{\prime \prime}$ pixels, and is stepped by $90^{\prime \prime}$ in the east-west direction and $180^{\prime \prime}$ in the north-south direction) versus MDI (which has a plate scale of $2^{\prime \prime}$ in full-disk mode) does not allow modes even as high as $\ell=25$ to be adequately resolved. It is likely that as longer time series of data from newer, higher-resolution magnetograph instrumentation are assembled, energy spectra (such as those shown in Fig. 5) may change, especially at the higher end of the $\ell$ spectrum due to the better observations of finer scales of magnetic field.

Of interest too is the exponent of the power-law dropoff of these average spectra for $\ell$ values greater than the value of $\ell$ for which the energy peaks. We can see from the lower panel of Figure 5 that the energy spectra from the MDI diachronic map dataset and the evolving-flux synoptic-map dataset have different slopes (i.e., they have different 


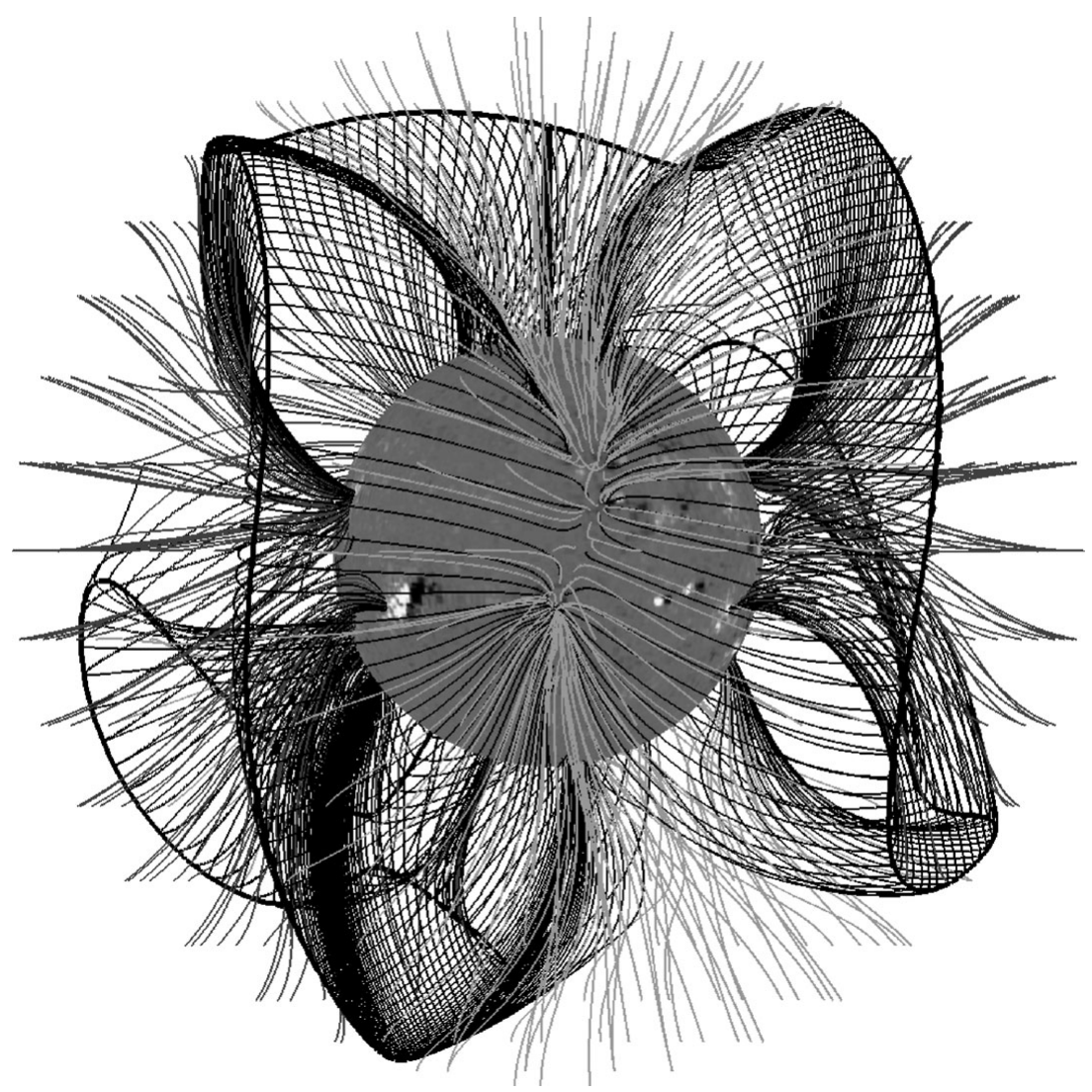

Figure 4. A potential-field source-surface (PFSS) model of the coronal magnetic field for $2000-O c t-11$, as an example of a time when the photospheric (and thus coronal) magnetic field has a quadrupolar component that dominates the dipolar component. The thin black lines correspond to high-arching closed field lines that abut the source-surface neutral line (thick black line). Some collections of such high-arching fieldlines, when viewed from a certain perspective, appear as "tunnels" that generally correspond to the location of helmet streamers seen in coronagraph imagery (e.g., Wang et al. 2007). Fieldlines intersecting the upper boundary of the model (which are assumed to open into the heliosphere) are colored either light or dark gray, depending on polarity.

power-law exponents), which must be an indication of the differences in the dynamics by which energy gets transferred from larger spatial scales to ephemeral-region scales in each of the two cases. Interestingly, the evolving-flux model was shown to have approximately the same flux distribution as a MDI full-disk magnetogram both at both low and high activity levels (see Fig. 2 of Schrijver 2001), making the differences between the model and the sun less obvious.

\section{Discussion and Concluding Remarks}

We have performed a spherical harmonic expansion on time series of photospheric magnetic field data assembled from multiple datasets, and illustrated several interesting features of the large-scale photospheric magnetic field. We find that both the polar and equatorial dipole components were much reduced during Cycle 23 than during the two preceding sunspot activity cycles. In contrast, the ratio of energies in the dipolar versus 

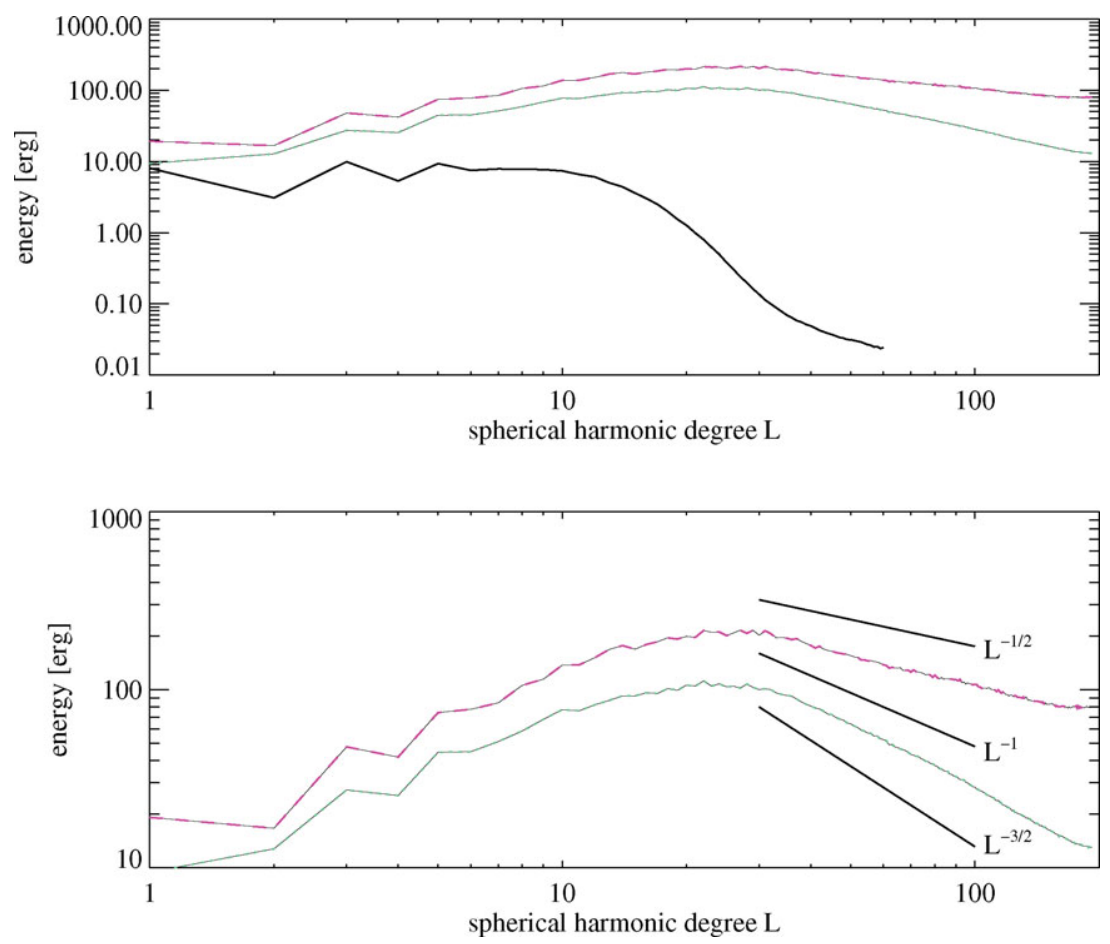

Figure 5. The energy spectra (upper panel) as a function of spherical harmonic degree $\ell$ as averaged over the full temporal extent of each dataset. The lower panel shows the same data for a portion of the vertical axis used in the upper panel. The solid, dashed, and dotted lines are as in Fig. 1.

the quadrupolar modes is similar, varying with about a factor of 10 between maximal and minimal activity levels during all three cycles.

During the past three sunspot activity cycles when the polar dipole is reversing sign, the quadrupolar modes were observed to predominate over the (both equatorial and polar) dipolar modes. Whether this feature plays a dynamical role in the reversal of the dipole, or whether it is simply a consequence of the polar dipole component being small (allowing higher-degree modes to dominate), remains at issue. We note in passing that similar dominance by the quadrupolar over the dipolar modes is observed during reversals of the geomagnetic field, and indeed interplay between these low-degree modes have been hypothesized to trigger polarity reversals for the earth's magnetic field (McFadden et al. 1991; Leonhardt \& Fabian 2007).

We also must keep in mind that the most detailed observations of photospheric magnetic fields occurred during the most recent sunspot cycle (Cycle 23), and that this particular sunspot activity cycle appears somewhat unusual (though not necessarily unprecedented) in many respects (Hoeksema 2010). For instance, the asymmetric nature of the distribution of active regions during this cycle resulted in one of the polar caps forming about one year prior to the other, and such dynamics may affect the type of large-scale diagnostics discussed in this article. Additionally, the upcoming Cycle 24 has also been slow to start up, resulting in an extended decline phase of Cycle 23, a fact that has confounded efforts to predict the length and strength of Cycle 24 (see, for example, the range of predictions compiled by Pesnell 2008). It is clear that improved observations 
over the course of upcoming cycles will help to explain some of the unsolved mysteries presented here.

\section{Acknowledgement}

A.S.B. acknowledges funding by the ERC through grant STARS2 \#207430.

\section{References}

Browning, M. K., Miesch, M. S., Brun, A. S., \& Toomre, J. 2006, ApJL, 648, L157

Charbonneau, P. 2005, Liv. Rev. Solar Phys., 2, 2

Hathaway, D. H. 2010, Liv. Rev. Solar Phys., 7, 1

Hoeksema, J. T. 2010, in A. G. Kosovichev, A. H. Andrei, \& J.-P. Roelot (eds.), Solar and Stellar Variability: Impact on Earth and Planets, Proc. IAU Symposium 264 (Cambridge: Cambridge Univ. Press), p. 222

Leonhardt, R. \& Fabian, K. 2007, Earth Planet. Sci. Lett., 253, 172

McFadden, P. L., Merrill, R. T., McElhinny, M. W., \& Lee, S. 1991, JGR, 96, 3923

Pesnell, W. D. 2008, Solar Phys., 252, 209

Scherrer, P. H. et al. 1995, Solar Phys., 162, 129

Schrijver, C. J. 2001, ApJ, 547, 475

Schrijver, C. J. \& De Rosa, M. L. 2003, Solar Phys., 212, 165

Wang, Y., Biersteker, J. B., Sheeley, Jr., N. R., Koutchmy, S., Mouette, J., \& Druckmüller, M. 2007, ApJ, 660, 882

\section{Discussion}

BRANDENBURG: If the quadrupole becomes dominant over the dipole, this should mean that Hale's polarity law is completely violated.

DeRosa: Axel, I believe you are referring to the fact that the $Y_{2,0}$ mode, when aligned with the axis of rotation, suggests that both poles should have flux possessing the same polarity, which would seem to violate the aspect of Hale's polarity law that indicates that the northern and southern hemispheres should be antisymmetric. However, I note that the quadrupole does not necessarily have to be aligned with the axis of rotation, and can in fact be tilted $90^{\circ}$ so as to be oriented in the equatorial plane. This is exactly what is occurring at the time there is more energy in the quadrupolar modes than in the dipolar modes [c.f., Fig. 4 in this article].

J. ToOmRe: What may be the relation between active longitudes and the predominance of quadrupole vs dipole modes?

DeRosa: The active longitudes, depending where they are, play a prominent role in determining which modes predominate, especially if they are regularly spaced. If they are separated by, say, $180^{\circ}$ and there is activity in the northern hemisphere at one of the active longitudes, and in the southern hemisphere at the other active longitude, then this puts power in the dipolar mode, for example. If there are more than two active longitudes, I would expect there to be persistent power in the higher-order spherical harmonics as a result. 